\title{
CASCA DA MANGA COMO FONTE DE MATÉRIA PRIMA LIGNOCELULÓSICA PARA OBTENÇÃO DO BIOETANOL
}

\section{MANGO PEEL AS A SOURCE OF LIGNOCELLULOSIC RAW MATERIAL FOR OBTAINING BIOETHANOL}

B. E. CARLOS ${ }^{1, *}$, K. E. EGÍDIO ${ }^{1}$, M. N. de M. OLIVEIRA ${ }^{2}$, C. R. C. de SOUSA ${ }^{3}$ e S. K. S. CARMO ${ }^{2}$

${ }^{1}$ Universidade Federal Rural do Semi-Árido, discente do Curso Interdisciplinar em Ciência e Tecnologia, Pau dos Ferros, Rio Grande do Norte, Brasil

${ }^{2}$ Universidade Federal Rural do Semi-Árido, Departamento de Ciências Exatas e Naturais, Pau dos Ferros, Rio Grande do Norte, Brasil

${ }^{3}$ Universidade Federal Rural do Semi-Árido, Departamento de Engenharias e Tecnologias, Pau dos Ferros, Rio Grande do Norte, Brasil

${ }^{*}$ Corresponding author. Universidade Federal Rural do Semi-Árido, discente do Curso Interdisciplinar em Ciência e Tecnologia, Pau dos Ferros, Rio Grande do Norte, Brasil, Telefone: +5584996199974

e-mail address: breno.eduardo1999@hotmail.com (B. E. Carlos).

\begin{tabular}{|c|}
\hline A R T I C L E I N F O \\
\hline $\begin{array}{l}\text { Article history: } \\
\text { Received 2020-06-30 } \\
\text { Accepted 2020-10-20 } \\
\text { Available online 2020-10-20 }\end{array}$ \\
\hline $\begin{array}{l}\text { palavras-chave } \\
\text { Meio-ambiente } \\
\text { Reaproveitamento } \\
\text { Biocombustível } \\
\text { Semi-Árido }\end{array}$ \\
\hline $\begin{array}{l}\frac{k e y w o r d s}{\text { Environment }} \\
\text { Reuse } \\
\text { Biofuel } \\
\text { Semi-arid }\end{array}$ \\
\hline
\end{tabular}

\begin{abstract}
A B S T R A C T
The use of fossil fuels has increasingly intensified the emission of polluting gases into the atmosphere, thus, second generation ethanol, obtained by lignocellulosic raw material, appears as an alternative because it is considered clean energy. Biofuel can be produced from residues generated from fruit production, such as mango peel, which has a composition that is satisfactory for the production of bioethanol. This work had as objective, the production of fermentable sugars, from this raw material, for that, a factorial planning was carried out about the main variables of interest in the process, and the acid hydrolysis technique was used. It was possible to observe a $25.49 \%$ increase in the formation offermentable sugars compared to the fresh composition of the material under study.
\end{abstract}

R E S U M O

O uso de combustíveis fósseis tem intensificado cada vez mais a emissão de gases poluentes à atmosfera, dessa forma, o etanol de segunda geração, obtido por matéria prima lignocelulósica surge como alternativa por ser considerado uma energia limpa. $O$ biocombustível pode ser produzido a partir de resíduos gerados da fruticultura, como exemplo, a casca da manga, a qual possui uma composição satisfatória à produção do bioetanol. Este trabalho teve por objetivo, a produção de açúcares fermentescíveis, a partir desta matéria prima, para isso, foi realizado um planejamento fatorial acerca das principais variáveis de interesse no processo, e empregado a técnica da hidrólise ácida. Pôde-se observar, um aumento de 25,49\% na formação de açúcares fermentescíveis comparados a composição in natura da matéria em estudo. 


\section{INTRODUÇ̃̃̃O}

A matriz energética mundial apresenta uma grande dependência da utilização de combustíveis fósseis. O desenvolvimento e utilização maior dos transportes individuais acabaram intensificando a emissão de gases poluentes expelidos para atmosfera como o monóxido e dióxido de carbono. O mundo, hoje, se depara com uma urgência na diversificação dessas formas de geração de energia. Daí aparece o bioetanol produzido através da matéria prima lignocelulósica como uma alternativa a esses combustíveis (SILVA, 2014).

A matéria-prima lignocelulósica é compreendida como resíduos agroindustriais. É formado a partir de celulose, lignina e hemicelulose, compostos encontrados em abundância na natureza (SILVA, 2014).

O Brasil é um grande produtor de frutas e pioneiro na América na produção da manga. Tornou-se um grande exportador do fruto e de seus subprodutos. A manga, por sua vez, apresenta uma estrutura constituída de três partes principais: casca, polpa e caroço. A casca e o caroço, que compreendem cerca de $40 \%$ do fruto, são desperdiçados em sua despolpagem. Essas parcelas, no entanto, são compostos que apresentam uma quantidade de celulose e hemicelulose interessantes à produção do bioetanol (COELHO et al., 2014).

Essa fabricação de bioetanol pode apresentar-se de diferentes formas e utilizando-se de várias técnicas, uma delas é a hidrólise ácida. Consistindo, basicamente, na conversão dos açúcares maiores presentes na biomassa em açúcares fermentescíveis, para posterior fermentação e obtenção do álcool (VIEGAS, 2013).

O trabalho, assim, tem como objetivo, a conversão de açúcares presentes na biomassa da casca da manga em açúcares menores, que são passíveis de fermentação. Além disso, buscase o aprimoramento da técnica da hidrólise ácida, avaliando os melhores parâmetros para realização dessa conversão.

\section{FUNDAMENTAÇÃO TEÓRICA}

\subsection{Bioetanol}

O bioetanol apresenta-se como uma proposta promissora no que diz respeito à geração e consumo de energia, em detrimento dos combustíveis fósseis, como o petróleo, que apresentam novas crises em relação à questão ambiental e econômica. Com o passar do tempo e o avanço dos estudos, houve uma intensificação na produção desse biocombustível a partir de resíduos lignocelulósicos (HENRIQUES, 2012).

Em vista disso, o bioetanol se destaca como um combustível que pode substituir os utilizados atualmente, principalmente porque é aplicável aos motores de combustão interna já existentes, diminuindo, dessa forma, a emissão de gases poluentes na atmosfera, como o dióxido de carbono (CO2) (HENRIQUES, 2012). Além de sustentável, também proporciona uma diminuição dos custos operacionais com o descarte do resíduo lignocelulósico, beneficiando agricultores e produtores (SILVA, 2014).

\subsection{Biomassa: casca da manga}

O Brasil é um grande produtor de frutas, chegando em 2014 a classificar-se como o terceiro maior do mundo (CARVALHO et al., 2017). No mercado brasileiro, o tipo de manga comercializado em maior quantidade é a variedade Tommy Atkins, conhecida popularmente como "Manga Rosa" (CORDEIRO, 2013).

O Nordeste é a principal região produtora de manga, correspondendo a $70 \%$ da produção nacional, chegando a obter em 2017 cerca de 812 mil toneladas da fruta (IBGE). O Rio Grande do Norte possui uma parcela de contribuição importante na produção da manga, colhendo, também em 2017, 2.816 hectares e tendo uma produção de 44.253 toneladas da fruta, contribuindo, assim, com $4 \%$ da produção nesse ano (IBGE).

O processamento da manga gera uma grande quantidade de resíduos, pois há o descarte completo do caroço e da casca da manga, quantificando de 28 a $43 \%$, que possui como componentes a celulose, lignina e hemicelulose, compostos essenciais à produção do bioetanol (CAVALCANTI et al., 2011).

\subsection{Hidrólise ácida}

A obtenção de bioetanol dá-se por diversas rotas, uma delas é a hidrólise ácida da matéria prima lignocelulósica. A hidrólise ácida se refere à quebra dos polissacarídeos da biomassa em açúcares fermentescíveis. Com essa transformação há também a remoção da lignina e pode-se seguir para fermentação alcoólica (BNDES, 2008). Essa técnica consiste, basicamente, no aquecimento da biomassa na presença de um ácido, que pode ser diluído ou concentrado. Dentre os ácidos mais utilizados para o processo, destaca-se o ácido clorídrico $(\mathrm{HCl})$ e o ácido sulfúrico $\left(\mathrm{H}_{2} \mathrm{SO}_{4}\right)$ (VIEGAS, 2013).

A composição da biomassa tem forte influência na eficiência da hidrólise ácida (BNDES, 2008). Dessa maneira, o indicado, em alguns casos, é a aplicação de um pré-tratamento, que consiste, simplesmente, em uma primeira hidrólise ácida, com o intuito de solubilizar a hemicelulose e tornar a estrutura cristalina da celulose mais suscetível às próximas hidrólises (ROCHA, 2016), retirando, também, a lignina, composto inibidor do processo fermentativo (SILVA, 2014).

Essa técnica apresenta-se bastante eficiente, pois pode atingir altas velocidades de reação, remoção quase completa das hemiceluloses na primeira etapa e um aumento na eficácia da hidrólise posterior da celulose (VIEGAS, 2013), com a quantidade de açúcares fermentescíveis obtidos podendo chegar a 90\% de eficiência quando há a aplicação de duas etapas (RODRIGUES, 2007). 


\section{MATERIAIS E MÉTODOS}

Inicialmente, o planejamento deste trabalho teve como ponto de partida a escolha da matéria lignocelulósica utilizada para transformação e obtenção de bioetanol. Após pesquisas realizadas, e por ter uma grande produção na região semiárida potiguar, onde é a localidade em que a pesquisa se desenvolve, foi escolhida a casca da manga, como fonte de celulose e hemicelulose, para conversão em bioetanol.

Para tal estudo foi determinado um planejamento fatorial sobre os testes a serem aplicados sobre a matéria prima, e para isto, 4 (quatro) parâmetros foram avaliados: tipo de ácido; concentração do ácido; temperatura e tempo. Estes parâmetros foram avaliados em uma etapa conhecida como pré-tratamento ácido, que se faz necessária para que a celulose apresente uma maior solubilidade nas próximas etapas.

Para o início do processo, foi realizada a secagem da matéria prima. A casca da manga in natura apresentada na Figura 1, obtida da região semiárida, foi distribuída sobre uma placa de alumínio revestida internamente com papel vegetal, e levada a uma estufa com circulação forçada de ar a uma temperatura de $105{ }^{\circ} \mathrm{C}$ por aproximadamente 3 (três) horas, realizando a pesagem a cada hora passada até obter-se uma massa constante.

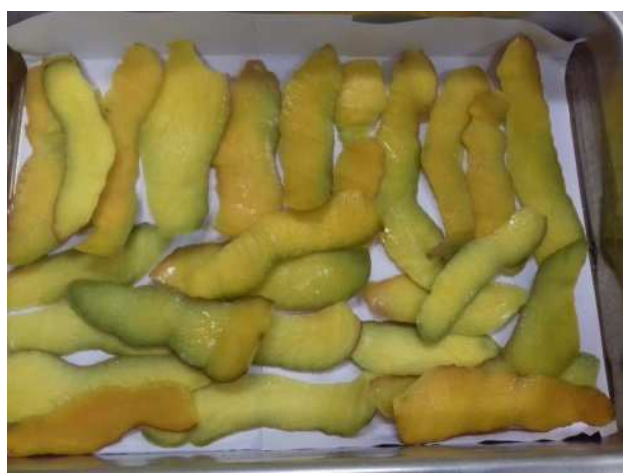

Figura 1 - Casca da manga in natura.

Depois, a casca da manga desidratada foi processada em um liquidificador comum até obter-se uma farinha bem fina, como mostra a Figura 2.

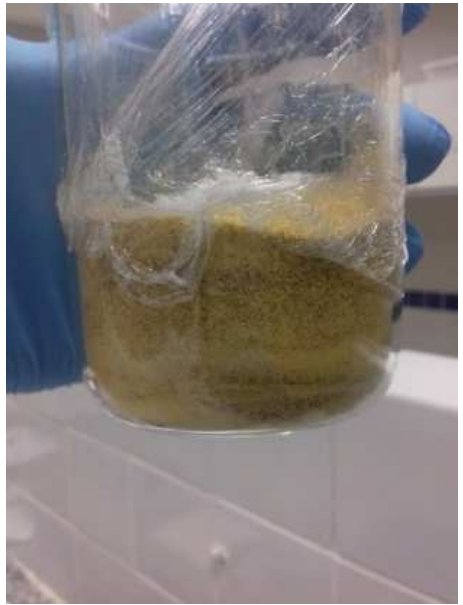

Figura 2 - Farinha da casca da manga (FMC)

\subsection{Planejamento fatorial}

Para os testes foi adotado um planejamento fatorial com base nas bibliografias consultadas. Com o planejamento fatorial é possível identificar a influência de cada parâmetro na etapa do processo. Dessa maneira, fixam-se três dos parâmetros explícitos e varia um desses, entendendo o comportamento da matéria-prima. Na Tabela 1, destacam-se os parâmetros adotados para os testes realizados no pré-tratamento.

Tabela 1 - Planejamento fatorial adotado para os testes.

\begin{tabular}{ccccc} 
Testes & Ácido & $\begin{array}{c}\text { Concentração } \\
\text { do ácido/ \% }\end{array}$ & $\begin{array}{c}\text { Tempe } \\
\text { ratura/ } \\
\text { o. }\end{array}$ & $\begin{array}{c}\text { Tempo/ } \\
\text { min }\end{array}$ \\
\hline $\mathbf{B R}$ & ÁGUA & -- & 28 & 10 \\
$\mathbf{1}$ & $\mathrm{HCl}$ & 0,05 & 100 & 120 \\
$\mathbf{2}$ & $\mathrm{HCl}$ & 0,1 & 100 & 120 \\
$\mathbf{3}$ & $\mathrm{HCl}$ & 0,5 & 100 & 120 \\
$\mathbf{4}$ & $\mathrm{HCl}$ & 1 & 100 & 120 \\
$\mathbf{5}$ & $\mathrm{HCl}$ & 5 & 100 & 120 \\
$\mathbf{6}$ & $\mathrm{HCl}$ & 10 & 100 & 120 \\
$\mathbf{7}$ & $\mathrm{H}_{2} \mathrm{SO}_{4}$ & 0,05 & 100 & 120 \\
$\mathbf{8}$ & $\mathrm{H}_{2} \mathrm{SO}_{4}$ & 0,1 & 100 & 120 \\
$\mathbf{9}$ & $\mathrm{H}_{2} \mathrm{SO}_{4}$ & 0,5 & 100 & 120 \\
$\mathbf{1 0}$ & $\mathrm{H}_{2} \mathrm{SO}_{4}$ & 1 & 100 & 120 \\
$\mathbf{1 1}$ & $\mathrm{H}_{2} \mathrm{SO}_{4}$ & 5 & 100 & 120 \\
$\mathbf{1 2}$ & $\mathrm{H}_{2} \mathrm{SO}_{4}$ & 10 & 100 & 120 \\
\hline
\end{tabular}

Assim, foi adicionada em um ernlenmeyer, $1 \mathrm{~g}$ de biomassa para $15 \mathrm{~mL}$ de ácido, escolhendo-se essa proporção com base no que foi estudado na revisão bibliográfica realizada. Esse frasco foi levado à banho-maria em uma das temperaturas estipuladas no planejamento fatorial em um tempo específico. Depois, o licor foi filtrado e armazenado em frascos, como mostra a Figura 3.

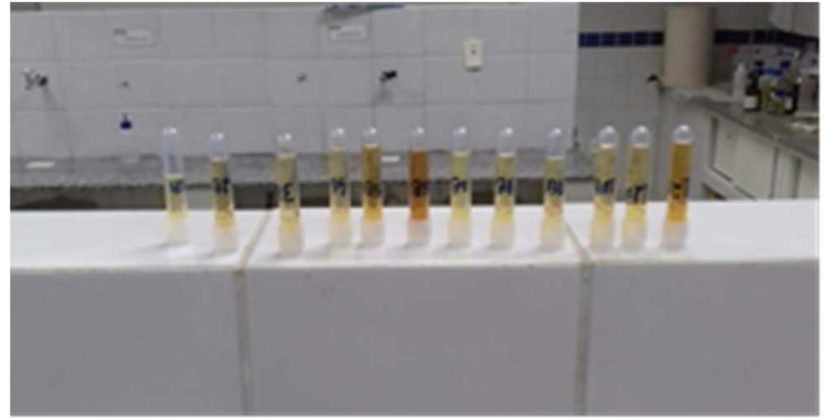

Figura 3 - Licores obtidos após o pré-tratamento ácido.

\subsection{Análise dos açúcares redutores totais (ART)}

Para a análise de ART, utilizou-se o método DNS (ácido 3,5-dinitrosalicílico), segundo metodologia obtida por Miller (1959), que apresenta a conversão de açúcares após as etapas de hidrólise. Dessa maneira, utiliza-se $1 \mathrm{~mL}$ do licor obtido no prétratamento ácido com $1 \mathrm{~mL}$ do DNS, seguindo uma metodologia específica. Essa mistura segue a banho maria e depois passa por uma diluição. Após a realização do procedimento, levam-se as amostras para leitura de absorbância (abs) por meio de um espectrofotômetro, conforme ilustrado na Figura 4, a seguir, em um comprimento de onda de $540 \mathrm{~nm}$. 


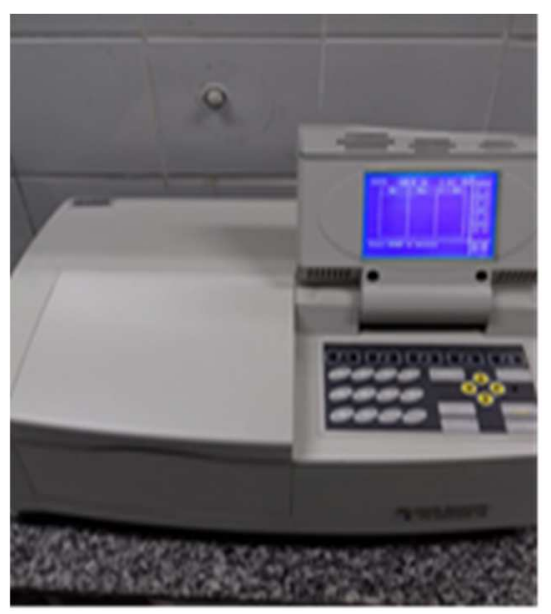

Figura 4 - Espectrofotômetro utilizado na medição da absorbância.

Essas medidas são convertidas em ART através da utilização de uma curva de calibração da glicose, que é elaborada tomando uma metodologia semelhante, modificando apenas o licor utilizado, que para curva são amostras com diferentes concentrações de glicose. Com os dados referentes a quantidade de açúcares desses licores de glicose, pode-se formar uma curva e obter a regressão linear desta. Os coeficientes da regressão fornecem os dados para o cálculo da quantidade de gramas de açúcares por litro de licor hidrolisado.

\section{RESULTADOS E DISCUSSÕES}

A Tabela 2 abaixo destaca os resultados obtidos na etapa de pré-tratamento da biomassa da casca da manga.

Tabela 2 - Resultados do pré-tratamento.

\begin{tabular}{cccccc} 
Teste & Ácido & $\begin{array}{c}\text { Conc./ } \\
\%\end{array}$ & $\begin{array}{c}\text { Temp. / } \\
{ }^{\circ} \mathbf{C}\end{array}$ & $\begin{array}{c}\text { Tempo/ } \\
\text { min }\end{array}$ & $\begin{array}{c}\text { Conc. } \\
\text { Açúcar / } \\
\text { g/L }\end{array}$ \\
\hline BR & ÁGUA & -- & 28 & 10 & 22,673 \\
$\mathbf{2}$ & $\mathrm{HCl}$ & 0,10 & 100 & 120 & 28,453 \\
$\mathbf{6}$ & $\mathrm{HCl}$ & 10 & 100 & 120 & 0,032 \\
$\mathbf{8}$ & $\mathrm{H}_{2} \mathrm{SO}_{4}$ & 0,10 & 100 & 120 & 27,008 \\
$\mathbf{1 2}$ & $\mathrm{H}_{2} \mathrm{SO}_{4}$ & 10 & 100 & 120 & $-0,006$ \\
\hline
\end{tabular}

Nos testes preliminares, foi possível obter uma boa conversão de açúcares em relação à quantidade presente inicialmente, denotada pelo teste BR. Nessa primeira avaliação, observou-se um aumento de $25,49 \%$ da quantidade de açúcares iniciais utilizando o $\mathrm{HCl}$ em concentração de $0,1 \%$. Para o $\mathrm{H} 2 \mathrm{SO} 4$ na concentração também de $0,1 \%$, foi possível obter um aumento de $19,12 \%$ da quantidade inicial.

A Tabela 2 também evidencia que para os testes iniciais de pré-tratamento, concentrações mais altas do ácido, na maioria das vezes, renderam menos açúcares, isto é, houve uma menor conversão ou houve a degradação de açúcares, gerando produtos de degradação que podem atrapalhar o processo fermentativo. Para provar tais suposições é necessário análises mais concisas, que serão realizadas posteriormente. Restringe-se, agora, ao melhor teste e deve-se trabalhar em cima dele para as etapas posteriores ao pré-tratamento ácido, no que tange as etapas de hidrólise ácida e fermentação alcoólica.

Os resultados aqui expostos são preliminares, avaliando apenas a possibilidade da utilização dessa biomassa para produção de bioetanol, sendo necessária a realização de mais testes para obtenção dos parâmetros adequados para essa produção. É importante ressaltar, ainda, que a maioria dos trabalhos leva rem consideração dois processos de hidrólise ácida, no entanto, para a matéria-prima em estudo não se obtiveram bons resultados com essas duas etapas.

\section{CONCLUSÃO}

De acordo com os resultados expostos, observou-se que o pré-tratamento ácido surtiu melhores resultados com a utilização de ácido clorídrico quando comparado ao outro ácido estudado. Foi possível ainda atestar, que a matéria-prima lignocelulósica adotada para estudo apresenta potencial para conversão de seus açúcares em açúcares fermentescíveis, uma vez que com a aplicação do processo houve um aumento de $25,49 \%$ na quantidade de açúcares iniciais. Assim, essa maior concentração de açúcares influencia nas etapas futuras, como a fermentação para obtenção do Bioetanol por destilação. Os testes preliminares denotam, dessa forma, o caminho a ser seguido para aprimoramento da técnica e maior conversão de açúcares a fim de produzir um combustível sustentável e de qualidade.

\section{AGRADECIMENTOS}

Agradecimentos a Universidade Federal Rural do SemiÁrido - UFERSA, Campus Pau dos Ferros e ao Grupo de Pesquisa em Processos e Análises Químicas - GPAQ.

\section{R E FER E N C I A S}

BIOETANOL de cana-de-açúcar: energia para o desenvolvimento sustentável. Rio de Janeiro: BNDES, 2008.

CARVALHO, C. de; KIST, B. B.; SANTOS, C. E. dos; TREICHEL, M.; FILTER, C. F. Anuário brasileiro da fruticultura 2017. Rio Grande do Sul: Editora Gazeta, 2017. ISSN 1808-4931.

CAVAlCANTI, M. T.; SIlva, V. C.; COSTA, T. S. da; FLORÊNCIO, I. M.; FLORENTINO, E. R. Obtenção do amido do endocarpo da manga para diversificação produtiva na indústria de alimentos. Revista Verde, Mossoró/RN, v. 6, n. 5, p. 80-83, dez. 2011.

COELHO, E. M.; VIANA, A. C.; AZEVÊDO, L. C. de. Prospecção tecnológica para o aproveitamento de resíduos industriais, com foco na indústria de processamento de manga. Cad. Prospec., Salvador/BA, v. 7, n. 4, p. 550-560, out./dez. 2014.

CORDEIRO, Edna Maria Silva. Biocompósitos poliméricos obtidos a partir da fração lignocelulósica e amilácea do caroço de manga (Mangifera indica), TOMMY ARKINS. 2013. Dissertação (Mestrado em Ciência e Engenharia dos Materiais), Universidade Federal do Rio Grande do Norte, Natal, RN, 2013. 
HENRIQUES, Henrique João Castanheiro. Viabilidade do uso de bioetanol como combustivel alternativo. 2012. Dissertação (Mestrado em Engenharia Mecânica), Universidade de Aveiro, Portugal, 2012.

ROCHA, Glauco Yves Gomes dos Santos. Hidrólise ácida do albedo de laranja lima. 2016. Dissertação (Mestrado em Energia da Biomassa), Universidade Federal de Alagoas, Alagoas, 2016.

RODRIGUES, Fábio de Ávila. Avaliação da tecnologia de hidrólise ácida de bagaço de cana. 2007. Dissertação (Mestrado em Engenharia Química) - Faculdade de Engenharia Química, Universidade Estadual de
Campinas, Campinas, SP, 2007.

SILVA, Rebeca de Almeida. Efeito do pré-tratamento ácido e básico na hidrólise enzimática do bagaço de acerola. 2014. Dissertação (Mestrado em Engenharia Química), Universidade Federal de Campina Grande, Campina Grande, PB, 2014.

VIEGAS, Mafalda Rosa. Avaliação de métodos de prétratamento na gasificação da biomassa. 2013. Dissertação (Mestrado em Energia e Bioenergia) Faculdade de Ciências e Tecnologia, Universidade Nova de Lisboa, Portugal, 2013. 